\title{
RESEARCH
}

Open Access

\section{Possible effects of EXT2 on mesenchymal differentiation - lessons from the zebrafish}

\author{
Malgorzata I Wiweger ${ }^{1,2}$, Carlos E de Andrea ${ }^{1,3}$, Karel W F Scheepstra' ${ }^{1}$, Zhe Zhao ${ }^{1,4}$ \\ and Pancras C W Hogendoorn ${ }^{1^{*}}$
}

\begin{abstract}
Background: Mutations in the EXT genes disrupt polymerisation of heparan sulphates (HS) and lead to the development of osteochondroma, an isolated/sporadic- or a multifocal/hereditary cartilaginous bone tumour. Zebrafish (Danio rerio) is a very powerful animal model which has shown to present the same cartilage phenotype that is commonly seen in mice model and patients with the rare hereditary syndrome, Multiple Osteochondroma (MO).
\end{abstract}

Methods: Zebrafish dackel (dak) mutant that carries a nonsense mutation in the ext2 gene was used in this study. A panel of molecular, morphological and biochemical analyses was used to assess at what step bone formation is affected and what mechanisms underlie changes in the bone formation in the ext2 mutant.

Results: During bone development in the ext $2^{-/-}$zebrafish, chondrocytes fail to undergo terminal differentiation; and pre-osteoblasts do not differentiate toward osteoblasts. This inadequate osteogenesis coincides with increased deposition of lipids/fats along/in the vessels and premature adipocyte differentiation as shown by biochemical and molecular markers. Also, the ext2-null fish have a muscle phenotype, i.e. muscles are shorter and thicker. These changes coexist with misshapen bones. Normal expression of runx2 together with impaired expression of osterix and its master regulator - xbp 1 suggest that unfolded protein responses might play a role in $\mathrm{MO}$ pathogenesis.

Conclusions: Heparan sulphates are required for terminal differentiation of the cartilaginous template and consecutive formation of a scaffold that is needed for further bone development. HS are also needed for mesenchymal cell differentiation. At least one copy of ext2 is needed to maintain the balance between bone and fat lineages, but homozygous loss of the ext2 function leads to an imbalance between cartilage, bone and fat lineages. Normal expression of runx2 and impaired expression of osterix in the ext $2^{-/-}$fish indicate that $\mathrm{HS}$ are required by osteoblast precursors for their further differentiation towards osteoblastic lineage. Lower expression of $x b p 1$, a master regulator of osterix, suggests that HS affect the 'unfolded protein response', a pathway that is known to control bone formation and lipid metabolism. Our observations in the ext2-null fish might explain the musculoskeletal defects that are often observed in $\mathrm{MO}$ patients.

Keywords: Zebrafish, Heparan sulphate, Bone, Fat, Osteochondroma, Exostosis, MHE/HME, Osteoblasts, Differentiation, Bone tumour

\footnotetext{
* Correspondence: p.c.w.hogendoorn@lumc.nl

${ }^{1}$ Department of Pathology, Leiden University Medical Center, Leiden, The Netherlands

Full list of author information is available at the end of the article
}

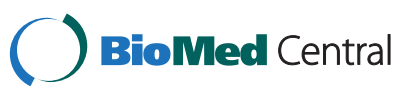

(c) 2014 Wiweger et al.; licensee BioMed Central Ltd. This is an Open Access article distributed under the terms of the Creative Commons Attribution License (http://creativecommons.org/licenses/by/2.0), which permits unrestricted use, distribution, and reproduction in any medium, provided the original work is properly credited. The Creative Commons Public Domain Dedication waiver (http://creativecommons.org/publicdomain/zero/1.0/) applies to the data made available in this article, unless otherwise stated. 


\section{Introduction}

Bone formation and homeostasis are complex processes in which many cell types and various signalling pathways are involved. Chondrocytes and osteoblasts originate from the same precursors - mesenchymal stem cells, which can also differentiate towards adipocytes, fibroblasts, myoblasts and epithelial cells. The osteoblast lineage is under strict control of RUNT-RELATED TRANSCRIPTION FACTOR 2 (RUNX2) and its downstream target - SP7/OSTERIX, which can work in a Run $x$-dependent and/or an independent manner. Run $x 2$ and Osterix mRNA are expressed in the immature chondro/osteoprogenitor cells and osteoblasts only [1] and alterations in the expression of either of the two genes affect bone development. Runx- and Osterix-null mice have normal cartilage but their reduced or absent expression of type I collagen, bone sialoprotein, osteonectin, osteopontin, and osteocalcin indicate that bone development is blocked at the step of pre-osteoblast to osteoblast differentiation [2]. Mammalian cells treated with an Osterix inhibitor, dexamethasone, enter an adipogenic- instead of osteoblastic lineage [3]. This imbalance between bone and fat is a known phenomenon. For example, it has been shown that knockout mice, which are heterozygous for Peroxisome proliferatoractivated receptor $\gamma$ (PPAR $\gamma$ have impaired adipogenesis, coinciding with an increased osteoblast number [4]. Other signalling molecules such as wingless (Wnt), bone morphogenic protein (BMP), and hedgehog were also shown to trigger the switch between different lineages including a bone-to-fat change. Remarkably, in all of these pathways, receptor-ligand binding and gradient formation is dependent on heparan sulphates (HS).

Heparan sulphate (HS) are glycosaminoglycans, heavily sulphated linear polysaccharides, that are present in all type of cells. Once they become attached to a core protein they form proteoglycans. The biosynthesis of HS take place in the Golgi apparatus and endoplasmic reticulum, where the elongation of glycosaminoglycan chains is maintained by type II glycosyltransferases encoded by the EXOSTOSINs genes, EXT1 and EXT2 [5]. Several genes are involved in the biosynthesis and degradation of HS, and mutations affecting the HS production have serious consequences. Abnormal accumulation of HS, due to its impaired degradation, causes mucopolysaccharidosis, a progressive disorder affecting mental and physical abilities, causing damage to various organs and leading to premature death. Patients with mucopolysaccharidosis often display skeletal abnormalities such as short stature or abnormal bone density [6,7]. Decreased levels of HS due to mutations in EXT1 or EXT2 also lead to a skeletal abnormality resulting in one of the most common benign bone tumours in young adults - osteochondroma [8]. The hereditary form of osteochondroma, multiple osteochondromas (MO; previously named multiple hereditary exostosis, MHE or hereditary multiple exostosis, HME), is a syndrome that is characterized by the development of multiple tumours (osteochondromas) at different sites of the endochondral skeleton [9]. MO is also associated with various other skeletal and non-skeletal phenotypes such as short stature, bone bowing (Figure 1), impingement of tendons, muscles or nerves as well as low bone density, lipid deposition within osteochondromas, pain and scarring [9-13].

Several mice models have been developed to study the role of EXT1 or EXT2 in bone and osteochondroma formation [14]. Zebrafish (Danio rerio) have also been shown to be a powerful animal model with morphological and developmental pathways comparable to those seen in humans [15]. We use zebrafish dackel (dak) mutants that carry a nonsense mutation in the ext2, gene

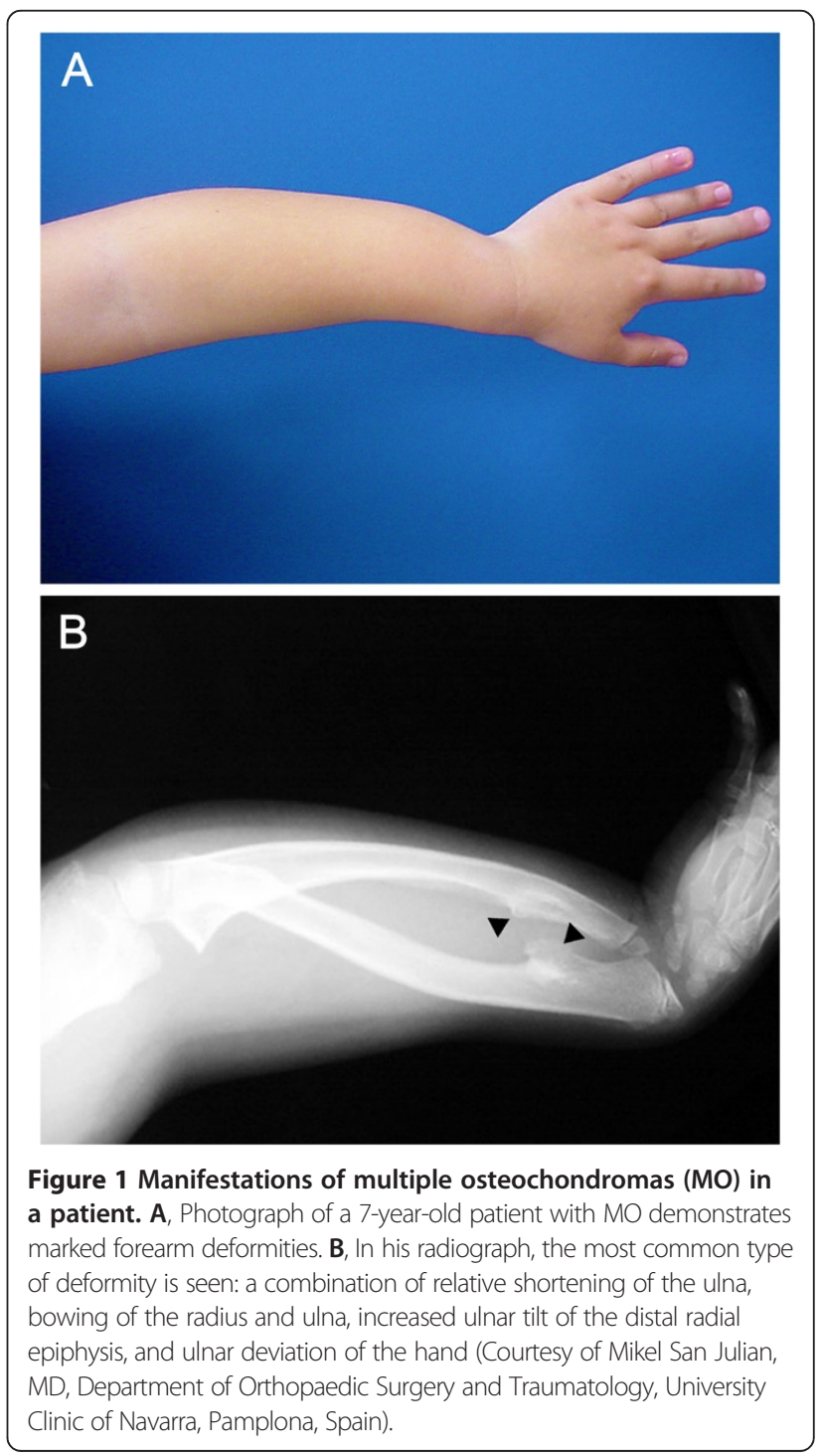


which is $84.7 \%$ identical (at protein level) with human EXT2 [16]. The ext $2^{-/-}$fish have been used as a model for MO. They have shown to mimic the cartilage phenotype (organization and behaviour) that is common to all models and the dental phenotype present in a number of patients but never described in mice [17-20].

In this study we show that bone development in the zebrafish $e x t 2^{-/-}$mutant is affected at two levels/stages in osteogenesis. Firstly development of the scaffold that is needed for osteoblasts to generate the bone is delayed/absent because chondrocytes fail to undergo terminal differentiation. Secondly, bone formation fails to progress from pre-osteoblasts towards osteoblasts and this change coexists with abnormal lipid depositions and premature adipocyte differentiation. Compounds stimulating fat-to-bone shift, GW9662 and purmorphamine, stimulate bone development in WT and ext2 heterozygote but do not rescue the ext2-null bones. Reduced expression of $x b p 1$, the master regulator of osterix, suggests that unfolded protein responses might play an important role in $\mathrm{MO}$ pathogenesis. Beside the "low bone-high fat phenotype", the ext2-null fish also have a muscle phenotype, i.e. muscles are shorter and thicker, and therefore might have different mechanical properties. Bone bowing, weak muscles and muscle fatigue are often observed in MO patients. Based on our findings in the fish model we speculate that bone bowing may occur as a result of weaker "fat bones" being distorted by muscles (with different mechanical properties). In support of this concept misshaped clavicles and bowed Meckel's are a very frequent phenomenon in the ext2-null mutant fish (data not shown).

\section{Materials and methods}

Animals

All experiments on zebrafish were performed in accordance with national and institutional guidelines for the care and use of laboratory animals. Zebrafish (Danio rerio $\mathrm{H}$.) $\mathrm{AB}$, golden and albino strains were used as wild type (WT) lines. Homozygote dackel (dak, ext $2^{\text {to273b }}$ ), knypek (kny, $\left.g p c^{u 34.8}\right)$, pinscher (pic, slc35b2 $\left.2^{14 M X}\right)$, hi307 ( $\beta 3$ gat $\left.3^{\text {hi307 }}\right)$ and hi954 (uxs1 $1^{\text {hi } 954}$ ) mutants were obtained in natural crosses and staged according to Kimmel et al. [21]. The dak mutant was also kept in a $T g$ (osteix:GFP) background [22]. Unless stated otherwise, embryos were anaesthetized in tricane, fixed in $4 \%$ paraformaldehyde, dehydrated in a series of methanol dilutions and stored at $-20^{\circ} \mathrm{C}$.

Bones were stained with Alizarin red as described previously [17]. Lipid deposits were visualized with Oil red $\mathrm{O}$ as described by Li and co-authors [23].

\section{Drug treatment}

Groups of 50 eggs were placed in a Petri dish with $20 \mathrm{ml}$ E3 medium. Prior to treatment fish larvae were manually decorionated. GW9662 (Sigma) at concentration of 5$20 \mu \mathrm{M}$ and/or purmorphamine (Calbiochem) at concentrations of 2,5-20 $\mu \mathrm{M}$ were added at 48, 60, 72, and 96 hours post fertilization (hpf) directly into E3 in which larvae were grown. 2-40 $\mu \mathrm{M}$ SB431542 (Tocris Biotrend), 0.2-4 $\mu \mathrm{M}$ dorsomorphin, $1-10 \mathrm{ng} / \mathrm{ml}$ TGF- $\beta 3$ (Oncogene Sci.), or 10-2500 ng/ml BMP6 (a gift from Dr. K. Sampath, Curis, Cambridge, MA) were added into E3 from $48 \mathrm{hpf}$. For control, equal volume of DMSO (solvent) was added. In case of TGF- $\beta 3$ and BMP6 activators, as a solvent and control, $4 \mathrm{mM} \mathrm{HCl}$ and $0.1 \%$ BSA were used. At 6 days post fertilization (dpf) fish were analysed for osterix expression (fish with transgenic osterix:GPP in the background) and for bone calcification (Alizarin red).

\section{In situ hybridization and immunohistochemistry}

Whole mount mRNA in situ was done accordingly to Thiesse 2008 [24] using: fabp11a, fabp11b and pparg rybo-probes. For amplification of the probe templates following primers were used: fabp11a_F 5'-GATCAAATC TCAATTTACAGCTGTTG-3', fabp11a_R + T7 5' -TAAT ACGACTCACTATAGGGTTCAAAGCACCATAAAGAC TGATAAT-3', fabp11b_F 5'-AACACTTTGTGCTATT ATCTGTC-3', fabp11b_R + T7 5'-TAATACGACTCACT ATAGGGCCATCCGCAAGGCTCATAG-3', pparg_F2 5'-TGCAGAGAACAGCGTTTCAT-3' and pparg_R1+ T7 5' -TAATACGACTCACTATAGGGCACTTCGATGA CCCCGTACT-3'. Whole mount immunostaining on zebrafish embryos was performed as described previously [17] using as a primary antibodies from the Developmental Studies Hybridoma Bank: anti-MF-20 for muscles and collagen II for cartilage, both in dilution of 1:250. For light microscopy, the anti-Digoxigenin-AP, Fab fragments (Roche) at 1:4000 or anti-mouse AP (Sigma) at 1:500 followed by BCIP/NBT (Sigma) were used to detect the signal. For confocal microscopy, Alexa 488 and 546 were used as the secondary antibody in dilution 1:200. Each experiment was repeated at least three times. Morphological evaluation was then performed by comparing of the ext2 homozygote mutant with its normal counterpart.

\section{Quantitative RT-PCR}

The expression levels of bone-, cartilage- and adipocytespecific markers were determined by quantitative real time PCR. RNA extraction was performed as described by de Jong and colleagues with on-column DNase I digestion [25]. cDNA synthesis was performed as described previously [26]. Possible genomic contamination in the cDNA preparations was tested by PCR using colla2 primers and confirmed as negative. The primer sets were designed using Primer3 online program. The sequences of the qPCR primers are listed in Table 1. Unless stated otherwise, the primers were designed as such 
Table 1 Sequences of primers used for quantitative PCR

\begin{tabular}{|c|c|c|c|}
\hline Primer name & Sequence $5^{\prime} \rightarrow 3^{\prime}$ & Lineage & Gene structure \\
\hline adopql aPCR F1 & AACCTGGAAGAGATGGCAGA & A & \\
\hline adopql aPCR R1 & CAGGAAAGCCTCTTGGTCCT & A & \\
\hline cebpa qPCR F1 & CACAACAGCTCCAAGCAAGA & A & \# \\
\hline cebpa $q P C R R 1$ & AATCCATGTAGCCGTTCAGG & A & \# \\
\hline cebpb qPCR F1 & TGTTCAGCCCGGACTTTATG & A & \# \\
\hline cebpb qPCR R1 & AGTCTGGTACGGCAGGTACG & A & $\#$ \\
\hline colla2 aPCR F2 & CTGGCATGAAGGGACACAG & B & \\
\hline colla2 qPCR R2 & GGGGTTCCATTTGATCCAG & B & \\
\hline col10a1 qPCR F2 & CCTGTCTGGCTCATACCACA & C & \\
\hline col10a1 aPCR R2 & AAGGCCACCAGGAGAAGAAG & C & \\
\hline osteocalcin qPCR F1 & TGAGTGCTGCAGAATCTCCTAA & B & \\
\hline osteocalcin $q P C R R 1$ & GTCAGGTCTCCAGGTGCAGT & B & \\
\hline osteopontin qPCR F1 & TGAAACAGATGAGAAGGAAGAGG & B & \\
\hline osteopontin $q P C R R 1$ & GGGTAGCCCAAACTGTCTCC & B & \\
\hline osterix $q P C R$ F2 & GCGTCGATTCTGGAGGAG & B & \\
\hline osterix $q P C R R 2$ & AATCTCGGACTGGACTGGTG & B & \\
\hline pparg $q P C R F 1$ & GGTTTCATTACGGCGTTCAC & A & \\
\hline pparg $q P C R R 1$ & TGCGGCTCTTCTTGTGTATG & A & \\
\hline runx2a $q P C R F 1$ & AACTTTCTGTGCTCGGTGCT & $B, C$ & \\
\hline runx2a $q P C R R 1$ & GTCATTTCCAGCCATTACCG & $B, C$ & \\
\hline run $\times 2 b q P C R F 2$ & CAAACACCCAGACCCTCACT & $B, C$ & \\
\hline$r u n \times 2 b a P C R R 2$ & GTATGACCATGGTGGGGAAG & $B, C$ & \\
\hline scdl $q P C R F 1$ & GTGGCGAAATGTCATTCTGA & A & \\
\hline scd1 $q P C R R 1$ & CCATACACGAAACACGCAAA & A & \\
\hline slc25a5 qPCR F1 & CCCCCATTGAGAGAGTCAAA & $\mathrm{HK}$ & \\
\hline slc25a5 qPCR R1 & ССTCTCCAGAACGACAGGAA & HK & \\
\hline sox9a qPCR F1 & GGAGCTCAGCAAAACTCTGG & C & \\
\hline sox9a aPCR R1 & AGTCGGGGTGATCTTTCTTG & C & \\
\hline srebp1c aPCR F1 & CCCCCAGCAGACTCTCTACA & A & \\
\hline srebp1c aPCR R1 & CGACAGACTCTGGATCGTCA & A & \\
\hline
\end{tabular}

$A$, adipocyte-specific gene; $B$, bone specific gene; $C$, cartilage specific gene; HK, house keeping gene. \#, gene with one exon.

Unless stated otherwise, the primers were designed to construct amplicons of 100-150 bp, spanning at least one intron to avoid amplification from possible genomic DNA contaminant in the template.

that the amplicons were $100-150 \mathrm{bp}$, spanning at least one intron. Tm was set at $60 \pm 1^{\circ} \mathrm{C}$. Quantitative real time PCR was carried out in BioRad iCycler system with SYBR Green SuperMix (BioRad), and was analysed with iCycler IQ (40 cycles, $1 \mathrm{~min} 95^{\circ} \mathrm{C}$ for denaturation and $1 \mathrm{~min} 60^{\circ} \mathrm{C}$ for annealing and elongation). All the samples were examined in duplicate or triplicate, and the expression of each marker was normalized to slc25a5 level. slc25a5 is one of a few house-keeping genes which, accordingly to our array data, is not differentially regulated in the ext $2^{-/-}$fish (unpublished).

\section{Lipid analysis}

For Oil red O stain, 6 days old fish were anesthetized in tricane and fixed in $4 \%$ paraformaldehyde for 1-3 hours at room temperature prior to 10 minutes incubation with the dye. After staining, fish were washed twice in PBS and sorted by phenotype to homozygote mutant and siblings. Oil red $\mathrm{O}$ was extracted from a group of 10 phenotyped fish by over-night incubation in 100\% methanol and quantified by measuring absorbance at $518 \mathrm{~nm}$.

For TLC analysis, 6 days old fish were anaesthetized in tricane, sorted in groups of 20 fish. WT, siblings or 
homozygote mutants were ground with a plastic pestle in a mixture of chloroform:methanol $(2: 1, \mathrm{v} / \mathrm{v})$ and incubated at room temperature for 15 minutes. To $1 \mathrm{ml}$ of extract $300 \mu \mathrm{l}$ of water was added. Samples were quickly vortexed at $2000 \mathrm{rpm}$ for 5 minutes. Bottom phase was washed twice with $0.5 \mathrm{ml}$ of water to be finally reduced in a speed-vacuum. Concentrated lipid extracts were spotted on a Silica gel 60 TLC plate (Merck). Plates were developed in a mixture of chloroform-ethanol-watertriethylamine $(30: 35: 7: 35, \mathrm{v} / \mathrm{v} / \mathrm{v} / \mathrm{v})$, sprayed with primuline and viewed under ultraviolet light.

\section{Statistical analysis}

Data are given as mean \pm standard error of mean (SEM). One sample t-test for comparing column means to a hypothetical value or two samples unpaired Student's test for comparison of two groups were used to determine statistical significance and described as * for $\mathrm{p}<0.05$, ** for $\mathrm{p}<0.005$ and ${ }^{* * *}$ for $\mathrm{p}<0.001$.

\section{Results}

Impaired bone development in the ext $2^{-/-}$fish has been described previously [17-19]. In order to identify at what step bone formation is affected and what mechanisms underlie changes in the ext 2 mutant, we examined the expression of various bone molecular markers in the ext $2^{-/-}$ fish and compared it with its siblings (Figure 2).

The importance of HS for pre-osteoblast differentiation Our previous mRNA in situ analyses demonstrated normal expression patterns of collagen 2 , sox $9 a$, and chondromodulin in the ext $2^{-/-}$fish, but did not give good estimates of the expression levels of these molecules $[17,18]$. In this work, using real time PCR, we confirm that in the homozygote ext 2 mutants, the expression levels of early skeletal markers such as runx2 are maintained at wild-type-levels whereas late skeletal markers such as osterix, collagen1a1, osteopontin and osteocalcin are approximately 2-fold down-regulated and collagen 10a1 shows even greater reduction (Figure 2B). Gene expression data indicate that HS are needed by chondrocytes for terminal differentiation for providing a scaffold for developing bone, and for maintenance of the osteochondroprogenitors/preosteoblasts to osteoblastic lineage.

Bone loss coincides with elevated lipid levels, premature adipocyte differentiation and misshapen musculature in the ext $2^{-1-}$ fish

Mesenchymal precursors can differentiate toward skeletalforming cells (osteoblasts and/or chondroblasts) and/or other lineages such as myoblasts and adipocytes [27]. Although differentiation of each lineage is controlled by multiple factors including HS-dependent hedgehog, Wnt or BMP, a switch in the fate of single or multiple lineages can be trigged relatively easily. Thus, we assessed whether diminished bone development in the ext $2^{-/-}$fish is compensated with gain of other lineage(s).

The whole mount MF-20-immunohistology revealed no obvious differences in the musculature between heterozygous ext 2 mutant and its wild type siblings (data not shown). However, the craniofacial muscles in the ext $2^{-/-}$fish were shorter, broader and fitted the misshapen cartilaginous skeleton (Figure 3). Moreover, some
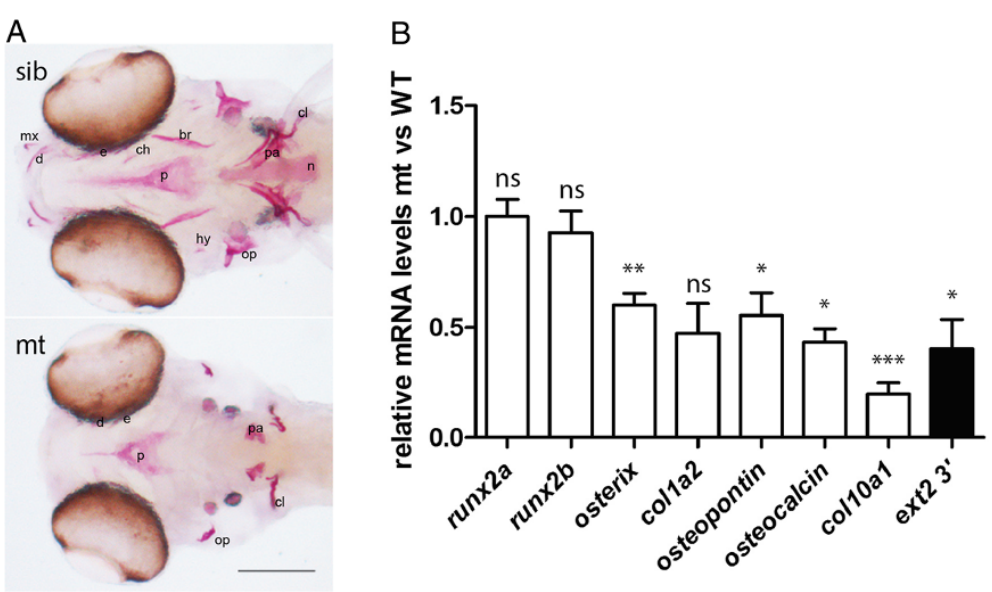

Figure 2 Bone development is impaired in the ext $\mathbf{2}^{-/-}$fish. A, Alizarin red stain for ossification in the craniofacial skeleton at $6 \mathrm{dpf}$. Dermal bones: dentary (d), maxilla (mx), parasphenoid (p), endopterygoid (e), branchiostegal rays (br), opercle (op), cleithrum (cl), cartilage bones: hyomandibula (hm), ceratohyal (ch). ceratobranchial 5/pharyngeal arch (pa); and notochord (n), scale = $0.1 \mathrm{~mm}$; $\mathbf{B}$, The relative change in the expression of bone markers at $5 \mathrm{dpf}$ was evaluated by real time PCR and analysed by delta-delta-Ct in the homozygote ext2 mutants vs. wild type. The results represent an average from minimum four single embryos. Expression was normalized against s/c25a5. Error bars indicate means with SEM. Expression of the ext2 was given as an example of a gene that was approximately 2-folds down-regulated and this under-expression was of biological relevance. 
muscles such as the hh were absent, whereas extra deposition of muscles was observed around ext $2^{-/-}$heart (Figure 3, Table 2, Additional file 1).

Oil red $\mathrm{O}$, a stain for neutral triglycerides, lipids and some lipoproteins, highlighted blood vessels, heart, tectum, guts, swim bladder and the remains of yolk in all fish (Figure 4A). In the ext $2^{-/-}$fish, the staining was intense and abnormally high lipid accumulation was observed. Especially, deposits in the vasculature were more pronounced (Figure 4A). Staining at the position of missing bones could be observed in some larvae. Significantly stronger $(\mathrm{P}<0.001)$ Oil red $\mathrm{O}$ stain in the ext $2^{-/-}$ fish coincided with over two fold overexpression of pparg (Figure 4). Other adipogenic markers such as cebp, srebp $1 c$ and $s c d 1$ were expressed at levels similar to wild type (Figure 4C). Despite intense staining, abnormal

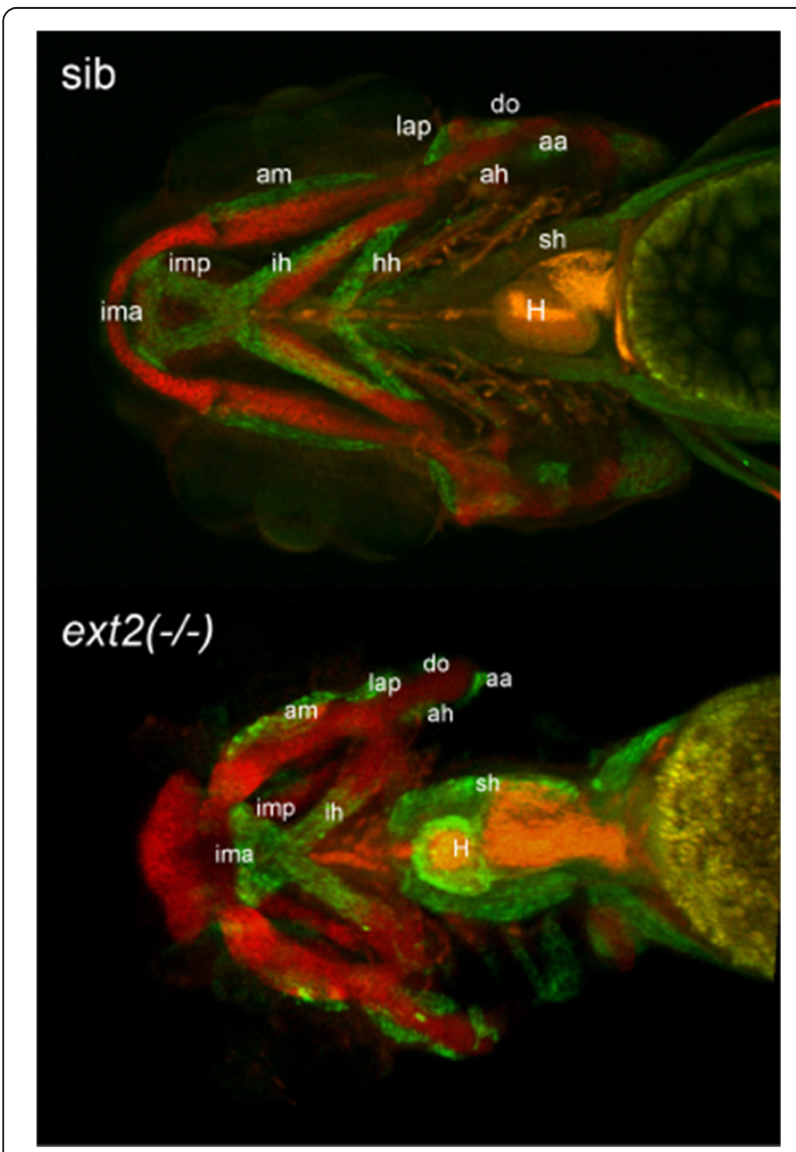

Figure 3 Homozygous ext2 mutant displays musco-skeletal phenotype. Whole mount immunolocalisation at 4dpf using MF-20 antibody for muscles (green) and collagen II for cartilage (red) shows thicker and shorter muscles fitting the malformed cartilaginous skeleton in the ext2 $2^{-1}$ fish. Muscles: intermandibularis anterior (ima), intermandibularis posterior (imp), adductor mandibulae (am), interhyoideus (ih), hyohyoideus (hh), levator arcus palatine (lap), adductor hyoideus (ah), dilator operculi (do), sternohyoideus (sh) and adductor operculi (ao). Note missing hh muscle and, marked with a star, increased musculature around heart $(H)$ in ext2-/-. Scale $=0.1 \mathrm{~mm}$. accumulation of lipids and overexpression of pparg, TLC analysis of lipid extracts did not reveal any changes in the profiles from wild type and ext $2^{-/-}$fish (data not shown).

Zebrafish adipocytes start to form by $8 \mathrm{dpf}$ and only upon feeding [29]. Interestingly, in the ext $2^{-/-}$fish, the mRNA in situ hybridization showed that fabp11a-expressing cells are present in unfed larvae already at $5 \mathrm{dpf}$ (Figure 4D).

\section{Bone-to-fat switch in proteoglycan mutants}

Observing a disturbance in the differentiation of mesenchymal cell lineages, we wonder if this is specific to the ext 2 mutant, or to proteoglycan deficiencies in common. Using a panel of mutants described in previous studies $[18,20]$, we found that the hi954 (uxs1) mutant lacking various proteoglycans and with a mild bone phenotype did not show any alteration in lipid deposition as judged by Oil red $\mathrm{O}$ (Figure $4 \mathrm{~B})$. Significantly increased $(\mathrm{P}<$ 0.005 ) levels of lipids were detected in the knypek (kny, $g p c 4^{-/-}$) mutant, which lacks only a portion of HS and has a mild bone phenotype (Figure $4 \mathrm{~B}$ and Additional file 2) $[18,20]$. Interestingly, the pinscher (pic/slc35b2) mutant, which fails to sulphate different molecules (including HS) and has a stronger bone phenotype $[17,18]$, only showed a very small, but statistically significant increase in lipid levels $(\mathrm{P}<0.05)$.

\section{Can PPARG inhibition rescue bone formation in the ext $2^{-/-}$homozygote mutant?}

Several drugs are known to affect lipid metabolism and influence the bone-to-fat balance. Although it is unlikely to expect a strong effect on total lipid levels in the early stages of zebrafish development where the majority of lipids come from yolk, application of GW9662, the antagonist of PPARG, was shown to enhance bone differentiation in zebrafish larvae [30]. As expected, we found that treatment with $15 \mu \mathrm{M}$ GW9662 added at 60hpf did not have any significant effect on lipid levels (Figure 5A) but did enhance formation of cartilage and dermal bones in wild type and in the ext2 heterozygous mutant (Figure 5B). In the ext $2^{-/-}$fish, with the same treatment, enhanced GFP expression was noted in tg(osterix:gfp) larvae (data not shown) with improved ossification of the previously existing bones. Bones that normally do not develop in ext $2^{-/-}$mutants, responded only partially to the treatment with rescue and stimulated ossification being observed in only some of the dermal bones; the ext $2^{-/-}$-cartilage bones were not rescued by this treatment (Figure 5B). Similar effects were seen upon $\geq 7.5 \mu \mathrm{M}$ purmorphamine treatment, which should stimulate a fat-tobone switch by activating hedgehog signalling (Figure 5). Furthermore, we tested involvement of other signaling pathways (HS-dependent) which stimulate bone-to-fat 
Table 2 Cranial muscles in zebrafish head

\begin{tabular}{|c|c|c|c|c|c|c|c|c|c|}
\hline \multirow[b]{2}{*}{ Region } & \multirow[b]{2}{*}{ Time } & \multirow[b]{2}{*}{ Muscle: } & & \multicolumn{2}{|c|}{$3 \mathrm{dpf}$} & \multicolumn{2}{|c|}{$4 \mathrm{dpf}$} & \multicolumn{2}{|c|}{$5 \mathrm{dpf}$} \\
\hline & & & & Wild type & $\operatorname{ext2}(-/-)$ & Wild type & $\operatorname{ext2}(-/-)$ & Wild type & ext2 (-/-) \\
\hline M & 62 & Intermandibularis anterior & ima & $x$ & $x^{*}$ & $x$ & $x$ & $x$ & $x$ \\
\hline $\mathrm{H}$ & 58 & Interhyoideus & ih & $x$ & $x$ & $x$ & $x$ & $x$ & $x$ \\
\hline B & 62 & Tranversus ventralis & tv & $x$ & $x$ & $x$ & $x$ & $x$ & $x$ \\
\hline M & 62 & Intermandibularis posterior & imp & $x$ & $x$ & $x$ & $x$ & $x$ & $x$ \\
\hline $\mathrm{H}$ & 58 & Hyohyoideus & hh & $x$ & a & $x$ & a & $x$ & a \\
\hline B & 85 & Rectus ventralis & rv & na & na & na & na & na & na \\
\hline B & 85 & Rectus communis & rc & na & na & na & na & na & na \\
\hline M & 53 & Adductor mandibulae & $\mathrm{am}$ & $x$ & $x$ & $x$ & $x$ & $x$ & $x$ \\
\hline M & 62 & Levator arcus palatini & lap & $x$ & $x$ & $x$ & $x$ & $x$ & $x$ \\
\hline M & 62 & Dilator operculi & do & $x$ & $x$ & $x$ & $x$ & $x$ & $x$ \\
\hline $\mathrm{H}$ & 68 & Adductor hyoideus & ah & $x$ & $x$ & $x$ & $x$ & $x$ & $x$ \\
\hline $\mathrm{H}$ & 68 & Adductor operculi & ao & $x$ & $x^{*}$ & $x$ & $x^{*}$ & $x$ & $x^{*}$ \\
\hline B & 72 & Dorsal pharyngeal wall & dpw & na & na & na & na & na & na \\
\hline $\mathrm{H}$ & 85 & Levator operculi & lo & na & na & na & na & na & na \\
\hline$E$ & 62 & Inferior oblique & io & na & na & na & na & na & na \\
\hline$E$ & 58 & Inferior rectus & ir & na & na & na & na & na & na \\
\hline E & 58 & Lateral rectus & Ir & na & na & na & na & na & na \\
\hline E & 53 & Medial rectus & $\mathrm{mr}$ & na & na & na & na & na & na \\
\hline B & 53 & Sternohyoideus & sh & $x$ & $x x$ & $x$ & $x x$ & $x$ & $x x$ \\
\hline E & 58 & Superior oblique & so & na & na & na & na & na & na \\
\hline$E$ & 58 & Superior rectus & sr & na & na & na & na & na & na \\
\hline $\mathrm{D}$ & 72 & Protractor pectoralis & $\mathrm{pp}$ & a & $x^{*}$ & $x$ & $x$ & $x$ & $x$ \\
\hline
\end{tabular}

Muscles were scored based on the MF-20 immuno staining of myosin (see Additional file 1) as described in [28] and indicated as: present (x), absent (a), or not analysed (na). Asterisk indicate severely reduced muscles. Minimum seven fish were analysed in each group. B, Branchail arches; D, Dorsal posterior; E, Extraocular; $\mathrm{H}$, Hyoid arch; and M, Manibular arch. Time - number of hours post fertilization at which a muscle become visible.

change. Treatment with BMP6 (an activator of BMP pathway) or dorsomorphine (an inhibitor of BMP) did not show significant effect at any time point on the craniofacial ext $2^{-/-}$bones and TGF- $\beta$ activator (TGF$\beta 3$ ligand) only partially stimulated dermal bones (data not shown).

\section{Is Ira1/Xbp1 pathway involved in the bone/lipid}

\section{phenotype of the ext2-/- fish?}

Recently, Xbp1 was shown to regulate osteoblast differentiation in a Runx2 independent manner [31]. Since in the ext2 $2^{-/}$fish the levels of runx2 transcript were normal while osterix levels were reduced, we wondered if the unfolded protein response is affected by the lack of HS. We found that heterozygotes maintained WT-levels of ern 1 and $x p b 1$. In the ext $2^{-/-}$mutant, the expression of ern1 was only slightly downregulated $\left(2^{\Delta \Delta} \mathrm{Ct}\right.$ ext $\left.2^{-/-} / \mathrm{WT}=0,71\right)$, but the expression of its downstream target, the $x b p 1$, was reduced to 0,64 .

\section{Discussion}

Abnormal lipid deposition coinciding with impaired bone formation is not common to all types of proteoglycan deficiencies (see Additional file 2). b3gat3- and uxs1-homozygote mutants, that are upstream of ext2 in the biosynthesis pathway and lack heparan and chondroitin sulphates, have a very mild bone phenotype and do not show increased lipid deposition (this work and data not shown). Interestingly, the fam $20 b$ and $x y l t 1$ mutants downstream of uxs 1 and upstream of b3gat 3 and ext2 were shown to have enhanced bone ossification [32]. Unfortunately nothing is known about fam $20 b$ and $x y l t 1$ lipid metabolism. The ext $2^{-/-}$and $g p c 4^{-/-}$, two mutants with reduced HS-levels only, have high lipid content; but only the ext 2 mutants have severely reduced bone formation, while the gpc4-null fish have very mild bone impairment. The slc35b2 homozygote mutant, which has diminished levels of all sulphated proteoglycans, has an even more severe bone phenotype than the ext $2^{-/-}$fish and show only very mild enhancement of lipid deposition. Why different proteoglycan deficiencies have such 


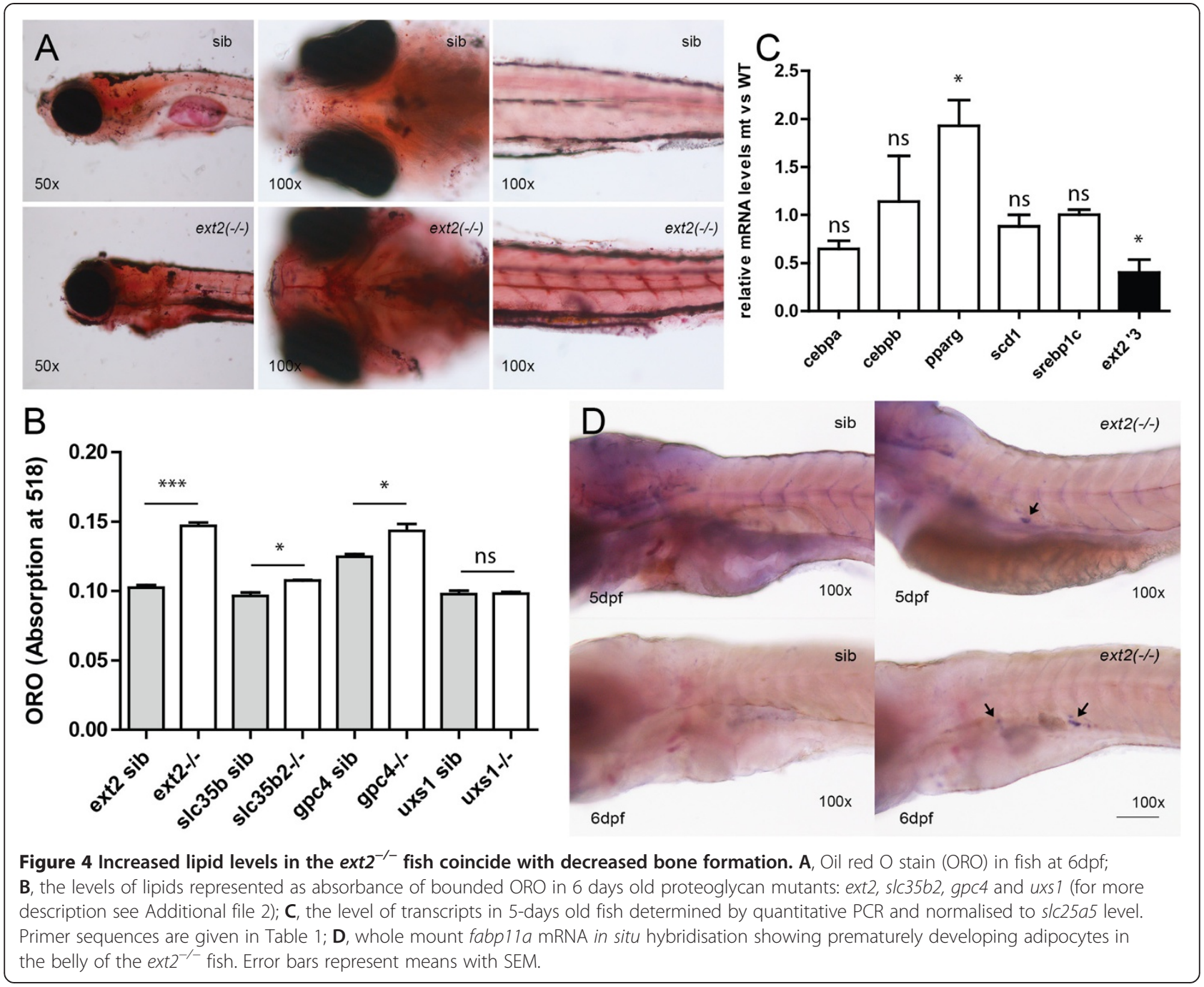

different effects on bone and lipid metabolisms is not clear. Holmborn and coauthors [33] showed that, in the ext 2 homozygote mutant, the remaining HS are oversulphated which changes their properties (i.e. increase occurrence of protein-interacting domains). Although, heparin, a highly sulphated glycosaminoglycan and a potent anticoagulant, which is often used in clinical practice, negatively affects bone density and is known to increase lipid deposition in sera, the role of over-sulphation of (proteo-)glycans would need to be confirmed.

Craniofacial skeletal development in zebrafish is of mixed origin being derived from cranial neural crest and/or mesoderm [34]. The presence of one functional copy of the ext2 gene is sufficient for the maintenance of normal differentiation of chondrocytes, osteoblasts and other mesenchyme-derived cells. Reduction in HS-levels in the ext $2^{-/-}$larvae clearly affects skeletal development. Loss of bones cannot be linked specifically to one type of precursor cell as both neural crest- and mesodermderived structures are affected. Despite their origin, two populations of osteoblasts with different sensitivity to hedgehog signalling have been described in zebrafish [35]. As no defects in the hedgehog signalling were found in the craniofacial skeleton of the ext $2^{-/-}$fish, it is unlikely that bone defects could be linked to a specific type of hedgehog-sensitive osteoblast. However, it is possible that there are multiple types of osteoblasts existing in fish, differing in their sensitivity for HS.

Bone homeostasis depends on the balance between osteoblastic and osteoclastic activity. Lipids are known to attract osteoclasts while suppressing osteoblastogenesis (for review see [36]). Unfortunately, we were not able to test this in zebrafish as the first osteoclasts develop by $16 \mathrm{dpf}$, beyond the time of premature death of the ext $2^{-/-}$ fish. Nevertheless, observations from patient material suggest that indeed both osteoblasts and osteoclasts are affected by HS-deficiencies $[10,26]$ or by HS abnormal accumulation [7] and, in both cases, bone mineral density is altered. Osteoblasts and adipocytes might not be the only lineages affected by imbalanced HS. EXT1-null 


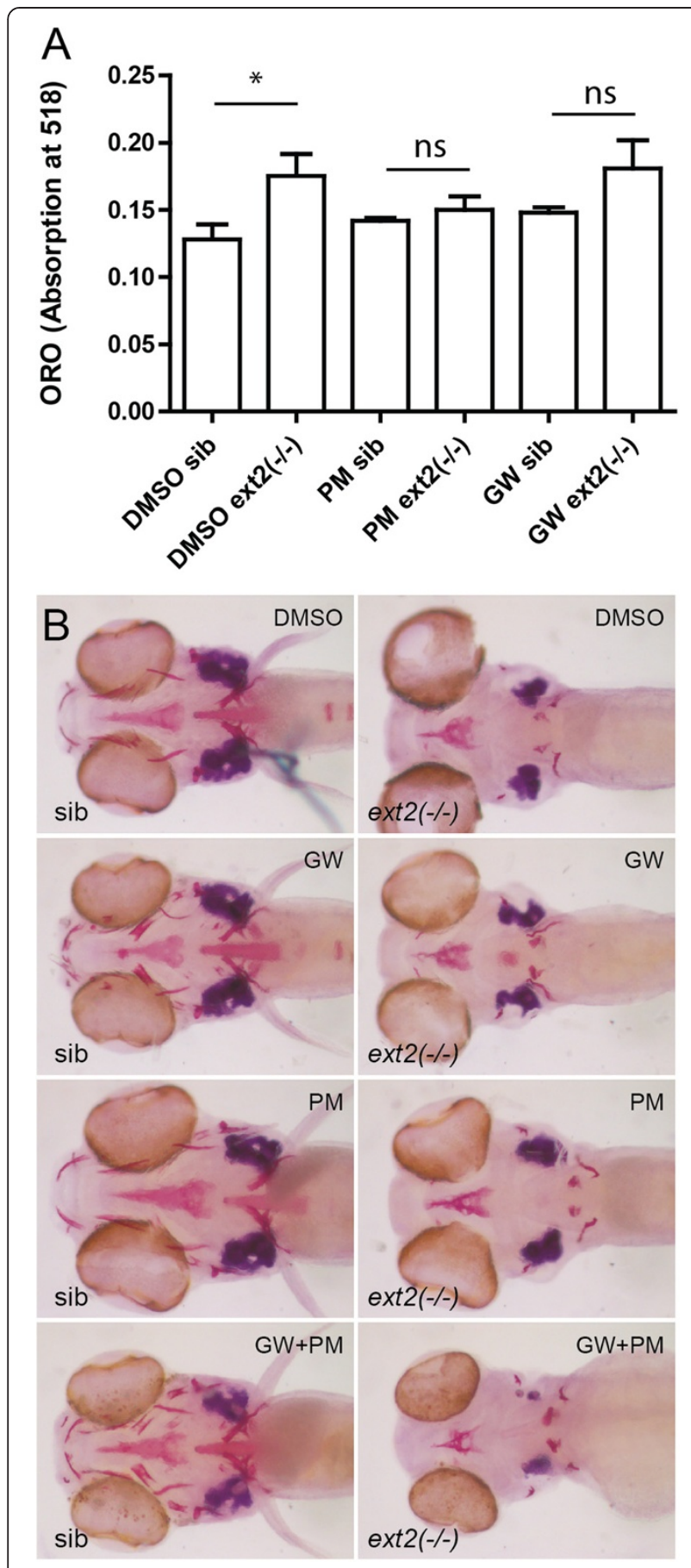

Figure 5 Bone and lipid phenotypes in the ext2 $2^{-/-}$fish are partially rescued by inhibition of PPAR or activation of hedgehog signalling. $\mathbf{A}$, Oil red $\mathrm{O}(\mathrm{ORO})$ stain for lipids in fish at 6dpf; B, Alizarin red stain for bones in fish at 6dpf. GW9662 (GW) and purmorphamine (PM) were added into fish water by $60 \mathrm{hpf}$ and fish were raised to $6 \mathrm{dpf}$. Minimum 10 fish were scored from each group and experiment was repeated with similar results at least three times. Error bars represent means with SEM. embryonic stem cells also appear to have impaired differentiation hematopoietic lineages [37], while osteochondromas exhibit impaired vascularisation [38].

Fatty acids, when not stored in adipocytes, accumulate into the circulation [39]. Although premature adipocytelike cells were detected in the ext2 $2^{-/-}$fish it is unlikely that they would be able to store all the lipids as cytoplasmic droplets. Therefore, Oil red $\mathrm{O}$ stain in vasculature could reflect only a surplus of fatty acids/lipids. However, it is also possible that mutation in the ext 2 gene leads to an abnormal intravascular accumulation of lipids. The changes in bones and fat that we have described in fish were a characteristic of an organism homozygous for a mutation in the ext2 gene in all cells. Since MO patients are mostly heterozygous for a mutation in EXT they should have very mild (if any) systemic phenotype. However, if findings from this fish model are true for humans, strong focal changes should be expected at the site where loss of heterozygosity/haploinsufficiency occured. Not much is known about lipid metabolism in patients with MO. Lemos and co-authors [10] reported lower bone mineral density of femoral neck and lumbar spine in MO patients near osteochondromas. In addition, single reports described deposition of fat within the cartilaginous cap of osteochondromas [11] and development of lipoma, a benign bone tumour, or fat-pads in association with osteochondromas [40,41]. These finding might have been coincidental in MO but increased lipid levels often remain asymptomatic. In light of our findings in the fish model on the bone-fat imbalance the status of lipids in human MO seems worth investigating.

Humans, mice and fish with MO are often short in stature and have bowed bones. Recently, Jones and coauthors [42] demonstrated that osteochondroma growing on account of deranged bone growth is apparent only in some individuals and other mechanisms must contribute to the short bone phenotype. Also bone bowing does not always require osteochondroma formation to generate the observed anatomical changes (K. Jones, University of Utah School of Medicine, personal communication). The presence of muscle phenotype needs to be confirmed in non-fish MO. Further work will show how (if) muscles with different mechanical properties contribute to the formation of shorter and bowed bones in patients.

\section{Conclusions}

Our data indicated that HS have multiple functions during endochondral bone development. First of all, HS are required for terminal differentiation of the cartilaginous template and consecutive formation of a scaffold that is needed for further bone development. Secondly, normal expression of runx2 and impaired expression of osterix 
in the ext $2^{-/-}$fish indicated that HS are required by osteoblast precursors for their further differentiation within the osteoblastic lineage. Furthermore, the increased lipid deposition in the ext $2^{-/-}$fish suggest that HS are involved in determining the cell lineage when mesenchymal precursor cell differentiates into bones and/or fat. PCR analyses confirm the increase in the expression of lipid markers and down-regulation of early skeletal markers. It still remains to be established how HS are involved in this shift, but lower expression of $x b p 1$, a master regulator of osterix, suggests that HS affect the unfolded protein response, a pathway which is known to control bone formation and lipid metabolism.

\section{Supporting data}

The data sets supporting the results of this article are included within the article and its additional files.

\section{Additional files}

Additional file 1: Muscle phenotype in the ext2 $2^{-/-}$fish. Muscles were detected with MF-20 antibody. Scale $=0.1 \mathrm{~mm}$.

Additional file 2: Information about affected proteoglycans and the bone- and fat phenotypes of mutants used in this study. Proteoglycans (PGS), heparan sulphate (HS), dermatan sulphate (DS), chondroitin sulphate (CS). keratan sulphate (KS) proteoglycans are the forth group of proteoglycan that is defected the $s / c 35 b 2^{-1-}$ mutant.

\section{Abbreviations}

AP: Alkaline phosphatase; b3gat3: Beta-1,3-glucuronyltransferase 3; BCIP/ NBT: 5-bromo-4-chloro-3-indolyl-phosphate/nitro blue tetrazolium; BMP: Bone morphogenetic proteins; BSA: Bovine serum albumine; dak: dackel; Dpf: Days post fertilization; ext1: exostosin 1; ext2: exostosin 2; HS: Heparan sulphates; HSPG: Heparan sulphate proteoglycan; Hpf: Hours post fertilization; kny: knypek; GFP: Green fluorescence protein; MO: Multiple osteochondromas; pic: pinscher; pparg: peroxisome proliferator-activated receptor gamma; runx2: runt-related transcription factor 2; s/c35b2: transport of adenosine 3'-phospho 5'- phosphosulfate (PAPS); TCL: Thin layer chromatography; TGF: Transforming/tumour growth factor; uxs 1: UDP-glucuronic acid decarboxylase 1; xbp 1: x-box binding protein 1.

\section{Competing interests}

All authors declare that they have no competing interests.

\section{Authors' contributions}

MIW, CEdA, KWFS, ZZ and PCWH designed this study, analyzed interpreted data; MIW, CEdA and KWFS collected data and generated figures. All authors were involved in writing the paper and had final approval of the submitted and published versions.

\section{Acknowledgments}

We thank Marcel Winter for the assistance with RT-PCR, Magnus Palmblad for shearing the lipid standards, Prof. van den Maren and his group for help with lipid analysis, Prof. Schulte-Merker for the Tg(osterix:gfp) zebrafish line, Dr. Mikel San Julian for sharing pictures of bowed bones, and Dr J.F. Graadt van Roggen for critical comments on the manuscript. The MF 20 antibody developed by D. Fischman was obtained from the Developmental Studies Hybridoma Bank (the auspices of the NICHD and maintained by The University of lowa, Department of Biology, lowa City, IA 52242). This work was supported by the European network of excellence EuroBoNeT [grant number 018814 (LSHC-CT-2006-018814)]

\section{Author details}

'Department of Pathology, Leiden University Medical Center, Leiden, The Netherlands. ${ }^{2}$ Current address: Zebrafish Core Facility, International Institute of Molecular and Cell Biology, Warsaw, Poland. ${ }^{3}$ Department of Histology and Pathology, University of Navarra, Pamplona, Spain. ${ }^{4}$ Nuffield Department of Medicine, Ludwig Institute for Cancer Research, University of Oxford, Oxford, UK.

Received: 18 October 2013 Accepted: 10 February 2014

Published: 14 March 2014

\section{References}

1. Kaback LA, Soung DY, Naik A, Smith N, Schwarz EM, O'Keefe RJ, Drissi H: Osterix/Sp7 regulates mesenchymal stem cell mediated endochondral ossification. J Cell Physiol 2008, 214:173-182

2. Nakashima K, Zhou X, Kunkel G, Zhang Z, Deng JM, Behringer RR, de Crombrugghe $B$ : The novel zinc finger-containing transcription factor osterix is required for osteoblast differentiation and bone formation. Cell 2002, 108:17-29.

3. Mikami Y, Lee M, Irie S, Honda MJ: Dexamethasone modulates osteogenesis and adipogenesis with regulation of osterix expression in rat calvaria-derived cells. J Cell Physio/ 2011, 226:739-748.

4. Akune T, Ohba S, Kamekura S, Yamaguchi M, Chung UI, Kubota N, Terauchi Y, Harada Y, Azuma Y, Nakamura K, Kadowaki T, Kawaguchi H: PPARgamma insufficiency enhances osteogenesis through osteoblast formation from bone marrow progenitors. J Clin Invest 2004, 113:846-855.

5. McCormick C, Leduc Y, Martindale D, Mattison K, Esford LE, Dyer AP, Tufaro F: The putative tumour suppressor EXT1 alters the expression of cellsurface heparan sulfate. Nat Genet 1998, 19:158-161.

6. Fung EB, Johnson JA, Madden J, Kim T, Harmatz P: Bone density assessment in patients with mucopolysaccharidosis: a preliminary report from patients with MPS II and VI. J Pediatr Rehabil Med 2010, 3:13-23.

7. Rigante D, Caradonna P: Secondary skeletal involvement in Sanfilippo syndrome. QJM 2004, 97:205-209.

8. Bovée JVMG, Heymann D, Wuyts W: Osteochondroma. In WHO Classification of Tumours of Soft Tissue and Bone. Edited by Fletcher CDM, Bridge JA, Hogendoorn PCW, Mertens F. Lyon: IARC; 2013:250-251.

9. Wuyts W, Bovée JVMG, Hogendoorn PCW: Multiple Osteochondromas. In WHO Classification of Tumours of Soft Tissue and Bone. Edited by Fletcher CDM, Bridge JA, Hogendoorn PCW, Mertens F. Lyon: IARC; 2013:384-385.

10. Lemos MC, Kotanko P, Christie PT, Harding B, Javor T, Smith C, Eastell R, Thakker RV: A novel EXT1 splice site mutation in a kindred with hereditary multiple exostosis and osteoporosis. J Clin Endocrinol Metab 2005, 90:5386-5392.

11. Schick F, Duda SH, Lutz O, Claussen CD: Lipids in bone tumors assessed by magnetic resonance: chemical shift imaging and proton spectroscopy in vivo. Anticancer Res 1996, 16:1569-1574.

12. Darilek S, Wicklund C, Novy D, Scott A, Gambello M, Johnston D, Hecht J: Hereditary multiple exostosis and pain. J Pediatr Orthop 2005, 25:369-376.

13. Hosalkar H, Greenberg J, Gaugler RL, Garg S, Dormans JP: Abnormal scarring with keloid formation after osteochondroma excision in children with multiple hereditary exostoses. J Pediatr Orthop 2007, 27:333-337.

14. Huegel J, Sgariglia F, Enomoto-Iwamoto M, Koyama E, Dormans JP, Pacifici M: Heparan sulfate in skeletal development, growth, and pathology: the case of hereditary multiple exostoses. Dev Dyn 2013, 242:1021-1032.

15. Dooley K, Zon L: Zebrafish: a model system for the study of human disease. Curr Opin Genet Dev 2000, 10:252-256.

16. Lee J-S, der HS V, Rusch MA, Stringer SE, Stickney HL, Talbot WS, Geisler R, Nüsslein-Volhard C, Selleck SB, Chien CB, Roehl H: Axon sorting in the optic tract requires HSPG synthesis by ext2 (dackel) and ext/3 (boxer). Neuron 2004, 44:947-960.

17. Clément A, Wiweger M, von der Hardt S, Rusch MA, Selleck SB, Chien CB, Roehl H: Regulation of zebrafish skeletogenesis by ext2/dackel and papst1/pinscher. PLoS Genet 2008, 4:e1000136.

18. Wiweger MI, Avramut CM, de Andrea CE, Prins FA, Koster AJ, Ravelli RB, Hogendoorn PC: Cartilage ultrastructure in proteoglycan-deficient zebrafish mutants brings to light new candidate genes for human skeletal disorders. J Pathol 2011, 223:531-542.

19. Wiweger MI, Zhao Z, van Merkesteyn RJ, Roehl HH, Hogendoorn PC: HSPGdeficient zebrafish uncovers dental aspect of multiple osteochondromas. PLoS One 2012, 7:e29734. 
20. de Andrea CE, Prins FA, Wiweger MI, Hogendoorn PC: Growth plate regulation and osteochondroma formation: insights from tracing proteoglycans in zebrafish models and human cartilage. J Pathol 2011, 224:160-168.

21. Kimmel CB, Ballard WW, Kimmel SR, Ullmann B, Schilling TF: Stages of embryonic development of the zebrafish. Dev Dyn 1995, 203:253-310.

22. Spoorendonk KM, Peterson-Maduro J, Renn J, Trowe T, Kranenbarg S, Winkler C, Schulte-Merker S: Retinoic acid and Cyp26b1 are critical regulators of osteogenesis in the axial skeleton. Development 2008, 135:3765-3774.

23. Li N, Felber K, Elks P, Croucher P, Roehl HH: Tracking gene expression during zebrafish osteoblast differentiation. Dev Dyn 2009, 238:459-466

24. Thisse C, Thisse B: High-resolution in situ hybridization to whole-mount zebrafish embryos. Nat Protoc 2008, 3:59-69.

25. de Jong M, Rauwerda H, Bruning O, Verkooijen J, Spaink HP, Breit TM: RNA isolation method for single embryo transcriptome analysis in zebrafish. BMC Res Notes 2010, 3:73.

26. Hameetman L, Rozeman LB, Lombaerts M, Oosting J, Taminiau AHM, Cleton-Jansen AM, Bovée JV, Hogendoorn PC: Peripheral chondrosarcoma progression is accompanied by decreased Indian Hedgehog signalling. J Pathol 2006, 209:501-511.

27. Takada I, Kouzmenko AP, Kato S: Wnt and PPARgamma signaling in osteoblastogenesis and adipogenesis. Nat Rev Rheumatol 2009, 5:442-447.

28. Schilling TF, Kimmel CB: Musculoskeletal patterning in the pharyngeal segments of the zebrafish embryo. Development 1997, 124:2945-2960.

29. Flynn EJ III, Trent CM, Rawls JF: Ontogeny and nutritional control of adipogenesis in zebrafish (Danio rerio). J Lipid Res 2009, 50:1641-1652.

30. Li N, Kelsh RN, Croucher P, Roehl HH: Regulation of neural crest cell fate by the retinoic acid and Pparg signalling pathways. Development 2010, 137:389-394.

31. Tohmonda T, Miyauchi Y, Ghosh R, Yoda M, Uchikawa S, Takito J, Morioka H, Nakamura M, Iwawaki T, Chiba K, Toyama Y, Urano F, Horiuchi K: The IRE1alpha-XBP1 pathway is essential for osteoblast differentiation through promoting transcription of Osterix. EMBO Rep 2011, 12:451-457.

32. Eames BF, Yan YL, Swartz ME, Levic DS, Knapik EW, Postlethwait JH, Kimme CB: Mutations in fam 20b and xylt1 reveal that cartilage matrix controls timing of endochondral ossification by inhibiting chondrocyte maturation. PLoS Genet 2011, 7:e1002246.

33. Holmborn K, Habicher J, Kasza Z, Eriksson AS, Filipek-Gorniok B, Gopal S, Couchman JR, Ahlberg PE, Wiweger M, Spillmann D, Kreuger J, Ledin J: On the roles and regulation of chondroitin sulfate and heparan sulfate in zebrafish pharyngeal cartilage morphogenesis. J Biol Chem 2012, 287:33905-33916.

34. Yelick PC, Schilling TF: Molecular dissection of craniofacial development using zebrafish. Crit Rev Oral Biol Med 2002, 13:308-322.

35. Hammond CL, Schulte-Merker S: Two populations of endochondral osteoblasts with differential sensitivity to Hedgehog signalling. Development 2009, 136:3991-4000.

36. Rosen CJ, Bouxsein ML: Mechanisms of disease: is osteoporosis the obesity of bone? Nat Clin Pract Rheumatol 2006, 2:35-43.

37. Holley RJ, Pickford CE, Rushton G, Lacaud G, Gallagher JT, Kouskoff V, Merry $C L$ : Influencing hematopoietic differentiation of mouse embryonic stem cells using soluble heparin and heparan sulfate saccharides. J Biol Chem 2011, 286:6241-6252.

38. de Andrea CE, Wiweger Ml, Bovee JV, Romeo S, Hogendoorn PC: Peripheral chondrosarcoma progression is associated with increased type $\mathrm{X}$ collagen and vascularisation. Virchows Arch 2012, 460:95-102.

39. Cousin W, Fontaine C, Dani C, Peraldi P: Hedgehog and adipogenesis: fat and fiction. Biochimie 2007, 89:1447-1453.

40. Ek ET, Slavin JL, Blackney MC, Powell GJ: Parosteal lipoma associated with an underlying osteochondroma arising from the hallux. Skeletal Radio 2007, 36:689-692.

41. Sakai H, Tamai K, Iwamoto A, Saotome K: Para-articular chondroma and osteochondroma of the infrapatellar fat pad: a report of three cases. Int Orthop 1999, 23:114-117.

42. Jones KB, Datar M, Ravichandran S, Jin H, Jurrus E, Whitaker R, Capecchi MR: Toward an understanding of the short bone phenotype associated with multiple osteochondromas. J Orthop Res 2013, 31:651-657.

doi:10.1186/1750-1172-9-35

Cite this article as: Wiweger et al:: Possible effects of EXT2 on mesenchymal differentiation - lessons from the zebrafish. Orphanet Journal of Rare Diseases 2014 9:35.

\section{Submit your next manuscript to BioMed Central and take full advantage of:}

- Convenient online submission

- Thorough peer review

- No space constraints or color figure charges

- Immediate publication on acceptance

- Inclusion in PubMed, CAS, Scopus and Google Scholar

- Research which is freely available for redistribution 